\title{
ON THE VIRTUAL WAITING TIME FOR AN M/G/1 RETRIAL QUEUE WITH TWO TYPES OF CALLS ${ }^{1}$
}

\author{
BONG DAE CHOI ${ }^{2}$ and DONG HWAN HAN \\ Korea Advanced Institute of Science and Technology \\ Department of Mathematics \\ Yusong-gu, Taejon 305-701, KOREA \\ GUENNADI FALIN \\ Moscow State University \\ Department of Probability, Mechanics and Mathematics Faculty \\ Moscow 119899, RUSSIA
}

\begin{abstract}
We consider an $M / G / 1$ retrial queueing system with two types of calls which models a telephone switching system. In the case that arriving calls are blocked due to the channel being busy, the outgoing calls are queued in priority group whereas the incoming calls enter the retrial group in order to try service again after a random amount of time. In this paper we find the Laplace-Stieltjes transform of the distribution of the virtual waiting time for an incoming call. When the arrival rate of outgoing calls is zero, it is shown that our result is consistent with the known result for a retrial queueing system with one type of call.
\end{abstract}

Key words: Retrial queues, virtual waiting time, Poisson process, Laplace-Stieltjes transform.

AMS (MOS) subject classifications: $\quad 60 \mathrm{~K} 25,60 \mathrm{~J} 20,90 \mathrm{~B} 22$.

\section{INTRODUCTION}

Retrial queueing systems are characterized by the feature that arriving calls who find the server busy join the retrial queue to try again for their requests in random order and at random intervals. Retrial queues have been widely used to model many problems in telephone switching systems, computer and communication systems. Simple examples in which retrials can be observed are telephone traffic and non-persistent CSMA protocol in the communication systems $[18,20]$. For comprehensive surveys of retrial queues, see Yang and Templeton [20] and Falin [10].

\footnotetext{
${ }^{1}$ Received: April, 1992. Revised: January, 1993.

${ }^{2}$ Supported by KOSEF 90-08-00-02.
} 
In this paper we consider a mathematical model of a telephone switching system. In modern telephone exchanges, subscriber lines are usually connected to the so-called subscriber line modules. These modules serve both incoming and outgoing calls. An important difference between these two types of calls lies in the fact that in the case of blocking due to all channels busy in the module, outgoing calls can be queued, whereas incoming calls get a busy signal and must be retried in order to establish the connection. As soon as the channel is free, an outgoing call, if present, occupies the channel immediately. Therefore incoming calls may not establish the connection as long as there are outgoing calls waiting. This fact implies that outgoing calls have non-preemptive priority over incoming calls.

Recently, Choi and Park [2] modeled the above system as an $M / G / 1$ retrial queue with two types of calls and derived the joint generating function of the number of calls in the two groups and the mean queue lengths. Khalil, Falin and Yang [15] investigated the same model with exponential multiple servers and obtained some of its properties such as the existence of a stationary regime and limit theorems under a high repetition intensity of blocked calls.

The main purpose of this paper, which is a continuation of [2], is to find the stationary distribution of the virtual waiting time for an incoming call. An $M / G / 1$ retrial queue with one type of calls is a special case of our model when the arrival rate of outgoing calls is zero. Falin and Fricker [11] calculated the Laplace-Stieltjes transform of the distribution of the virtual waiting time for ordinary $M / G / 1$ retrial queue with one type of call. For papers related to our model, see [13, 18]. We follow Falin and Fricker's technique [11] to derive the Laplace-Stieltjes transform of the distribution of the virtual waiting time.

This paper is organized as follows. In Section 2, we describe the model in detail. In Section 3, we derive the Laplace-Stieltjes transform of the distribution of the virtual waiting time for an incoming call. It is shown that our results are consistent with known results for a special case [11].

\section{THE MODEL}

As a mathematical model of a telephone switching system, we consider an $M / G / 1$ queue in which two independent Poisson flows arrive with rate $\lambda_{1}$ and $\lambda_{2}$. Calls from the Poisson flow with rate $\lambda_{1}$ (the Poisson flow with rate $\lambda_{2}$, respectively) can be identified as outgoing calls (incoming calls, respectively) in the telephone switching systems (see Figure 1). 
Incoming calls arrive at the system according to a Poisson process with rate $\lambda_{2}$. If an incoming call upon arrival finds the server free, he immediately occupies the server and leaves the system after service. If he finds the server busy on his arrival, he enters the retrial group in order to seek service again after a random amount of time. He persists this way until he succeeds the connection. The retrial time (the time interval between two consecutive attempts made by a call in the retrial group) is exponentially distributed with mean $1 / \nu$ and is independent of all previous retrial times and all other stochastic processes in the system.

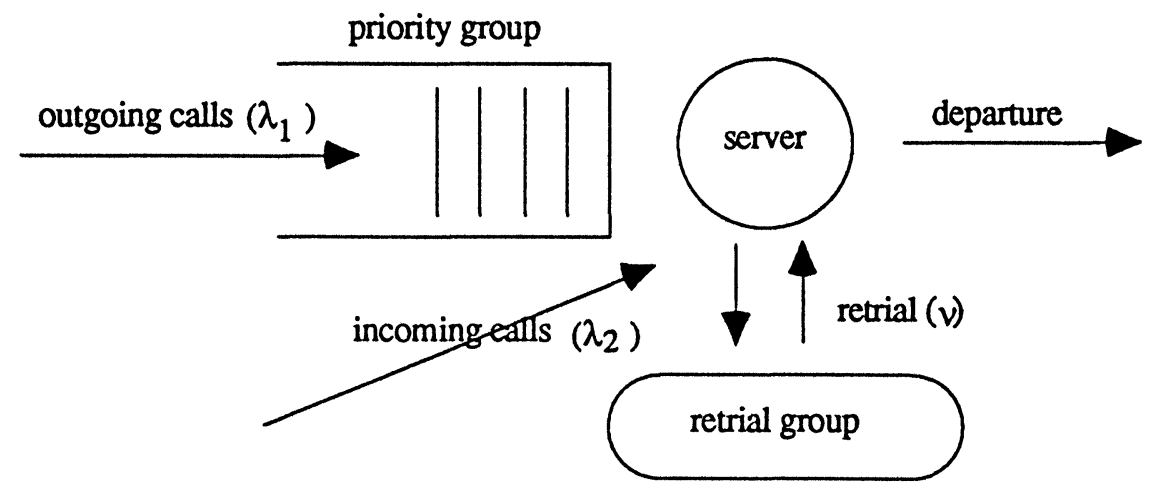

Figure 1.

The outgoing calls arrive at the system according to a Poisson process with rate $\lambda_{1}$. They are queued in a priority group after blocking and then are served in accordance with some discipline such as FIFO or random order. As soon as the server is free, an outgoing call occupies the server immediately, so incoming calls in the retrial group will be served only when there are no outgoing calls in the priority group. According to the above rule, outgoing calls in the priority group have non-preemptive priority over incoming calls.

We assume that the service times of both types of calls are independent and identically distributed with p.d.f. $b(x)$ and mean $b$. The assumption about the same distribution of two types of calls is analytically convenient and is practically natural in the sense that both incoming and outgoing calls in a telephone switching system have the same characteristic for service times. We need to assume that the service time has the density function to guarantee (13). Let

$$
b^{*}(\theta)=\int_{0}^{\infty} e^{-\theta x} b(x) d x
$$

be the Laplace transform of the p.d.f. of the service time. It is easy to show that the system is stable provided that $\rho=\lambda b<1$, where $\lambda=\lambda_{1}+\lambda_{2}$ (see $[2,12]$ ). We consider only stable 
systems in this paper.

Next we define the random variables;

$N_{1}(t)=$ the number of outgoing calls in priority group (excluding the call in service) at time $t$, $N_{2}(t)=$ the number of incoming calls in retrial group at time $t$, $X(t)=$ the residual service time of call in service at time $t$, $\xi(t)=\left\{\begin{array}{l}0, \text { when the server is idle at time } t, \\ 1, \text { when the server is busy at time } t .\end{array}\right.$

Define the probabilities;

$q_{j}(t)=P\left\{N_{2}(t)=j, \xi(t)=0\right\}$

$q_{i j}(t, x) d x=P\left\{N_{1}(t)=i, N_{2}(t)=j, X(t) \in(x, x+d x], \xi(t)=1\right\}$.

\section{THE VIRTUAL WAITING TIME}

The distribution of the waiting time of an outgoing call can be obtained from the theory of priority queueing systems [14]. In this paper, we consider only the distribution of waiting time for an incoming call.

First we introduce an auxiliary Poisson process $\left\{N_{c}(t), t \geq 0\right\}$ with intensity $s, s>0$, which is independent of the functioning of the system. The events of this Poisson process will be called 'catastrophes'. Name any arriving call as a tagged call. Suppose that the tagged incoming call arrives to the system and finds the server busy. Then he enters the retrial group. We name the first call, which departs the systems since the arrival of the tagged call, the 0-th call and then we number calls in their departing order (see Figure 2).

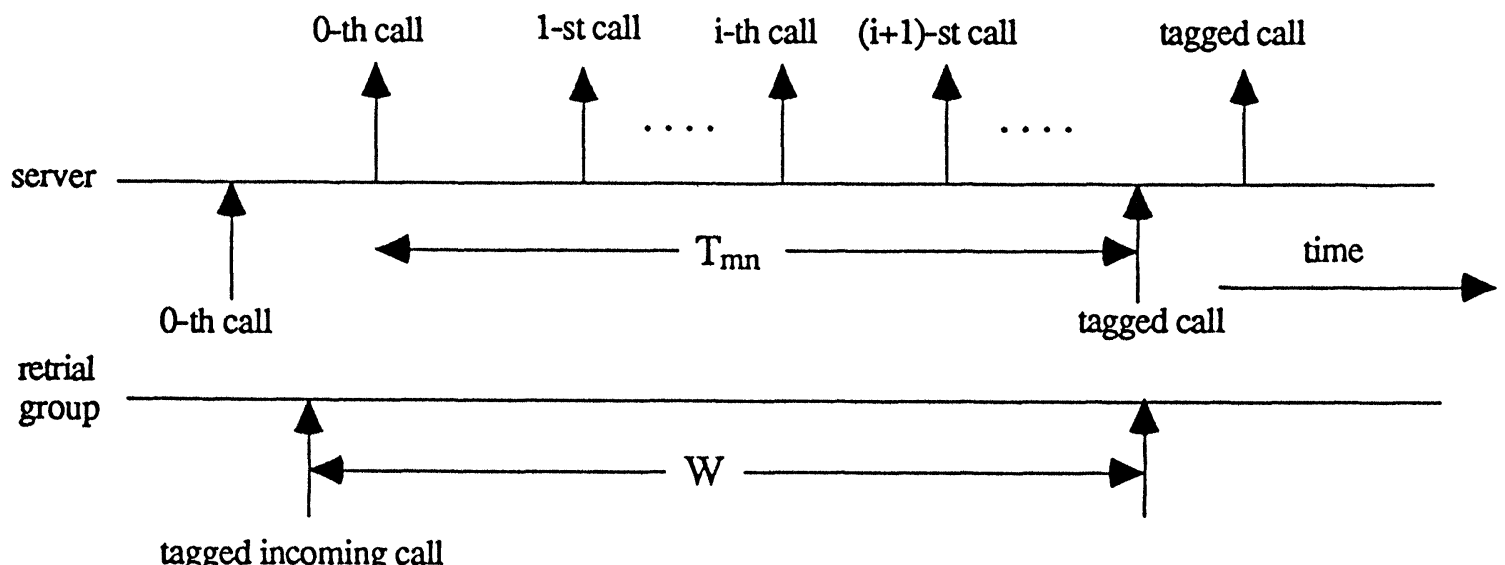

Figure 2. 
Suppose that at the moment of departure of the 0 -th call, there are $m$ outgoing calls and $n$ incoming calls. Let $p_{k l}^{(i)}(s)$ denote the probability that at the time of the $i$-th departure there are $k$ outgoing calls and $l$ incoming calls including the tagged call and until this time, no catastrophe occurs. The initial condition of $p_{k l}^{(i)}(s)$ is

$$
p_{k l}^{(0)}(s)=\left\{\begin{array}{lc}
1, & \text { if } k=m, l=n \\
0, & \text { otherwise }
\end{array}\right.
$$

Theorem 1: $\quad$ The probabilities $p_{k l}^{(i)}(s)$ are calculated from the following recursive formulas:

$$
\begin{aligned}
p_{k l}^{(i+1)}(s)= & \sum_{k^{\prime}=1}^{k+1} \sum_{l^{\prime}=1}^{l+1} p_{k^{\prime} l^{\prime}}^{(i)}(s) f_{k-k^{\prime}+1, l-l^{\prime}}(s) \\
& +\sum_{l^{\prime}=1}^{l+1} p_{0 l^{\prime}}^{(i)}(s)\left[\frac{\lambda}{s+\lambda+l^{\prime} \nu} f_{k, l-l^{\prime}}(s)+\frac{\left(l^{\prime}-1\right) \nu}{s+\lambda+l^{\prime} \nu} f_{k, l-l^{\prime}+1}(s)\right]
\end{aligned}
$$

where by convention

and

$$
f_{k,-1}(s)=0
$$

$$
f_{k l}(s)=\int_{0}^{\infty} e^{-s t} \frac{e^{-\lambda_{1} t}\left(\lambda_{1} t\right)^{k}}{k !} \frac{e^{-\lambda_{2} t}\left(\lambda_{2} t\right)^{l}}{l !} b(t) d t
$$

is the probability that during one service time, exactly $k$ outgoing calls and $l$ incoming calls arrive and no catastrophe occurs.

Proof: Let $t_{i}$ be the time of the $i$-th departure. Then

$$
\begin{gathered}
p_{k l}^{(i+1)}(s)=P\left[N_{1}\left(t_{i+1}\right)=k, N_{2}\left(t_{i+1}\right)=l, N_{c}\left(t_{i+1}\right)=0\right] \\
=\sum_{k^{\prime}=0}^{k+1} \sum_{l^{\prime}=1}^{l+1} p_{k^{\prime} l^{\prime}}^{(i)}(s) P\left[N_{1}\left(t_{i+1}\right)=k, N_{2}\left(t_{i+1}\right)=l, N_{c}\left(t_{i+1}\right)=0 \mid N_{1}\left(t_{i}\right)=k^{\prime}\right. \\
\left.N_{2}\left(t_{i}\right)=l^{\prime}, N_{c}\left(t_{i}\right)=0\right] .
\end{gathered}
$$

To find conditional probabilities

$$
P\left[N_{1}\left(t_{i+1}\right)=k, N_{2}\left(t_{i+1}\right)=l, N_{c}\left(t_{i+1}\right)=0 \mid N_{1}\left(t_{i}\right)=k^{\prime}, N_{2}\left(t_{i}\right)=l^{\prime}, N_{c}\left(t_{i}\right)=0\right]
$$

we have two cases depending on $k^{\prime}$. 
Case 1: $k^{\prime}=0$ and the $(i+1)$-st call to be served arrives before the first catastrophe occurs. This event is divided into two disjoint events depending on whether the $(i+1)$-st call is from an outside system or from a retrial group. First we treat the case that the $(i+1)$-st call to be served is a call (either outgoing or incoming) from outside the system. This even occurs with probability $\lambda /\left(s+\lambda+l^{\prime} \nu\right)$. In order that there are $k$ outgoing calls and $l$ incoming calls and no catastrophe occurs until the moment of the $(i+1)$-st call's departure, exactly $k$ outgoing calls and $l-l^{\prime}$ new incoming calls must arrive with no catastrophe occurring during the service time of the $(i+1)$-st call. The above event occurs with probability $f_{k, l-l^{\prime}}(s)$.

Next we treat the case that the $(i+1)$-st call to be served, is an incoming call (excluding the tagged call) from the retrial group. This event occurs with probability $\left(l^{\prime}=1\right) \nu /\left(s+\lambda+l^{\prime} \nu\right)$. By applying total probability law, we have

$$
\begin{aligned}
P\left[N_{1}\left(t_{i+1}\right)=\right. & \left.k, N_{2}\left(t_{i+1}\right)=l, N_{c}\left(t_{i+1}\right)=0 \mid N_{1}\left(t_{i}\right)=0, N_{2}\left(t_{i}\right)=l^{\prime}, N_{c}\left(t_{i}\right)=0\right] \\
= & \frac{\lambda}{s+\lambda+l^{\prime} \nu} f_{k, l-l^{\prime}}(s)+\frac{\left(l^{\prime}-1\right) \nu}{s+\lambda+l^{\prime} \nu} f_{k, l-l^{\prime}+1}(s) .
\end{aligned}
$$

Case 2: $\quad k^{\prime} \neq 0$. In this case, there are outgoing calls waiting in the priority group at the moment of the $i$-th departure, the next service immediately begins with an outgoing call in the priority group. In order that there are $k$ outgoing calls and $l$ incoming calls and no catastrophe occurs until the moment of the $(i+1)$-st call's departure, exactly $k-k^{\prime}+1$ outgoing calls and $l-l^{\prime}$ new incoming calls must arrive with no catastrophe occurring during the service time of the $(i+1)$-st call. Thus

$$
\begin{aligned}
P\left[N_{1}\left(t_{i+1}\right)\right. & \left.=k, N_{2}\left(t_{i+1}\right)=l, N_{c}\left(t_{i+1}\right)=0 \mid N_{1}\left(t_{i}\right)=k^{\prime}, N_{2}\left(t_{i}\right)=l^{\prime}, N_{c}\left(t_{i}\right)=0\right] \\
= & f_{k-k^{\prime}+1, l-l^{\prime}}(s) .
\end{aligned}
$$

For $z_{j}$, with $\left|z_{j}\right| \leq 1, j=1,2$,

$$
P^{(i)}\left(s, z_{1}, z_{2}\right)=\sum_{k=0}^{\infty} \sum_{l=1}^{\infty} p_{k l}^{(i)}(s) z_{1}^{k} z_{2}^{l}
$$

defines the joint generating function for $p_{k l}^{(i)}(s)$ with respect to $k$ and $l$.

From equation (1), we have

$$
\begin{aligned}
P^{(i+1)}\left(s, z_{1}, z_{2}\right)= & \frac{\beta\left(s, z_{1}, z_{2}\right)}{z_{1}}\left(P^{(i)}\left(s, z_{1}, z_{2}\right)-P^{(i)}\left(s, 0, z_{2}\right)\right) \\
& +\beta\left(s, z_{1}, z_{2}\right) \sum_{l^{\prime}=1}^{\infty} \frac{\lambda}{s+\lambda+l^{\prime} \nu} z_{2}^{l^{\prime}} p_{0 l^{\prime}}^{(i)}(s)
\end{aligned}
$$




$$
+\frac{\beta\left(s, z_{1}, z_{2}\right)}{z_{2}} \sum_{l^{\prime}=1}^{\infty} \frac{\left(l^{\prime}-1\right) \nu}{s+\lambda+l^{\prime} \nu} z_{2}^{l^{\prime}} p_{0 l^{\prime}}^{(i)}(s)
$$

where $\beta\left(s, z_{1}, z_{2}\right)=b^{*}\left(s+\lambda_{1}\left(1-z_{1}\right)+\lambda_{2}\left(1-z_{2}\right)\right)$.

Next we introduce the generating function for $P^{(i)}\left(s, z_{1}, z_{2}\right)$ with $|w| \leq 1$,

$$
P\left(s, z_{1}, z_{2}, w\right)=\sum_{i=0}^{\infty} P^{(i)}\left(s, z_{1}, z_{2}\right) w^{i} .
$$

We multiply both sides of (2) by $w^{i+1}$ and sum over $i$. Then we obtain

$$
\begin{gathered}
P\left(s, z_{1}, z_{2}, w\right)-z_{1}^{m} z_{2}^{n}=\frac{w \beta\left(s, z_{1}, z_{2}\right)}{z_{1}}\left(P\left(s, z_{1}, z_{2}, w\right)-P\left(s, 0, z_{2}, w\right)\right) \\
+w \beta\left(s, z_{1}, z_{2}\right) \sum_{i=0}^{\infty} w^{i} \sum_{j=1}^{\infty} \frac{\lambda}{s+\lambda+j \nu} p_{0 j}^{(i)}(s) z_{2}^{j} \\
+\frac{w \beta\left(s, z_{1}, z_{2}\right)}{z_{2}} \sum_{i=0}^{\infty} w^{i} \sum_{j=1}^{\infty} \frac{(j-1) \nu}{s+\lambda+j \nu} p_{0 j}^{(i)}(s) z_{2}^{j}
\end{gathered}
$$

This can be rewritten as

$$
\begin{gathered}
\left(z_{1}-w \beta\left(s, z_{1}, z_{2}\right)\right) P\left(s, z_{1}, z_{2}, w\right)=z_{1} w \beta\left(s, z_{1}, z_{2}\right) \sum_{i=0}^{\infty} w^{i} \sum_{j=1}^{\infty} \frac{\lambda}{s+\lambda+j \nu} p_{0 j}^{(i)}(s) z_{2}^{j} \\
+z_{1} w \beta\left(s, z_{1}, z_{2}\right) \sum_{i=0}^{\infty} w^{i} \sum_{j=1}^{\infty} \frac{(j-1) \nu}{s+\lambda+j \nu} p_{0 j}^{(i)}(s) z_{2}^{j-1} \\
+z_{1}^{m+1} z_{2}^{n}-w \beta\left(s, z_{1}, z_{2}\right) \sum_{i=0}^{\infty} w^{i} \sum_{j=1}^{\infty} p_{0 j}^{(i)}(s) z_{2}^{j} .
\end{gathered}
$$

Consider the function

$$
g\left(s, z_{1}, z_{2}, w\right)=z_{1}-w \beta\left(s, z_{1}, z_{2}\right) .
$$

For each fixed $s, z_{2}, w$, with $s>0,\left|z_{2}\right| \leq 1,|w| \leq 1$, regard $g\left(s, z_{1}, z_{2}, w\right)$ as a function of $z_{1}$. On the unit circle $\left|z_{1}\right|=1$, we see that $\operatorname{Re}\left(s+\lambda_{1}\left(1-z_{1}\right)+\lambda_{2}\left(1-z_{2}\right)\right)>0$. Using the well known fact that $\left|b^{*}(\theta)\right|<1$ for $\theta>0$, we must have $\left|\beta\left(s, z_{1}, z_{2}\right)\right|<\left|z_{1}\right|$ on $\left|z_{1}\right|=1$. By Rouchés theorem, there is a unique solution $z_{1}=\phi_{1}\left(s, z_{2}, w\right)$ of the equation $g\left(s, z_{1}, z_{2}, w\right)=0$ in the unit disc, i.e.,

$$
g\left(s, \phi_{1}\left(s, z_{2}, w\right), z_{2}, w\right)=\phi_{1}\left(s, z_{2}, w\right)-w \beta\left(s, \phi_{1}\left(s, z_{2}, w\right), z_{2}\right)=0
$$

As a matter of fact, $\phi_{1}\left(s, z_{2}, w\right)$ has the following explicit probabilistic meaning by Takács' lemma [5],

$$
\phi_{1}\left(s, z_{2}, w\right)=E\left[e^{-\left(s+\lambda_{2}\left(1-z_{2}\right)\right) L_{\infty} w^{I} \infty}\right]
$$


where $L_{\infty}$ is the length of a busy period and $I_{\infty}$ is the number of calls served during this period in the ordinary $M / G / 1$ queue with the arrival rate $\lambda_{1}$ and p.d.f. $b(x)$ of service times in the steady state. Furthermore

$$
\left|\phi_{1}\left(s, z_{2}, w\right)\right|<1
$$

for $|w| \leq 1$ and $\operatorname{Re}\left(s+\lambda_{2}\left(1-z_{2}\right)\right) \geq 0$ except $w=1$ and $s+\lambda_{2}\left(1-z_{2}\right)=0$ (see [5]).

Next we will eliminate $P\left(s, z_{1}, z_{2}, w\right)$ from the left-hand side of (3) and then obtain the relationship between the $p_{0 j}^{(i)}(s)$ 's which will be used to find the virtual waiting time. By inserting $z_{1}=\phi_{1}\left(s, z_{2}, w\right)$ into (3), we obtain

$$
\begin{aligned}
\phi_{1}\left(s, z_{2}, w\right)^{m} z_{2}^{n}=\sum_{i=0}^{\infty} w^{i} \sum_{j=1}^{\infty} p_{0 j}^{(i)}(s) z_{2}^{j}-\phi_{1}\left(s, z_{2}, w\right) \sum_{i=0}^{\infty} w^{i} \sum_{j=1}^{\infty} \frac{\lambda}{s+\lambda+j \nu} p_{0 j}^{(i)}(s) z_{2}^{j} \\
-\phi_{1}\left(s, z_{2}, w\right) \sum_{i=0}^{\infty} w^{i} \sum_{j=1}^{\infty} \frac{(j-1) \nu}{s+\lambda+j \nu} p_{0 j}^{(i)}(s) z_{2}^{j-1} .
\end{aligned}
$$

Let us introduce the following transformation

$$
\psi\left(s, z_{2}, w\right)=\sum_{i=1}^{\infty} w^{i} \sum_{j=1}^{\infty} \frac{1}{s+\lambda+j \nu} p_{0 j}^{(i)}(s) z_{2}^{j} .
$$

We will see from (9) that $\nu \psi(s, 1,1)$ is the probability that the tagged call occupies the server before the first catastrophe occurs. By (6) we have a singular first order differential equation for $\psi\left(s, z_{1}, w\right)$

$$
\begin{aligned}
\nu\left[\phi_{1}\left(s, z_{2}, w\right)-z_{2}\right] \frac{d \psi\left(s, z_{2}, w\right)}{d z_{2}} & \\
= & {\left[(s+\lambda)-\left(\lambda-\frac{\nu}{z_{2}}\right) \phi_{1}\left(s, z_{2}, w\right)\right] \psi\left(s, z_{2}, w\right)-\left[\phi_{1}\left(s, z_{2}, w\right)\right]^{m} z_{2}^{n} . }
\end{aligned}
$$

Define the function

$$
h\left(s, z_{2}, w\right)=z_{2}-\phi_{1}\left(s, z_{2}, w\right)
$$

For each fixed $s, w$ with real number $s>0,|w| \leq 1$, regard $h\left(s, z_{2}, w\right)$ as a function of $z_{2}$. Using (5) and Rouché's theorem, we have that there is a unique solution $z_{2}=\phi_{2}(s, w)$ of the equation $h\left(s, z_{2}, w\right)=0$ in the unit disc. It is easy to see that for real $s$ and $w$ with $s>0$, $0<w<1, \phi_{2}(s, w)$ is real and $0<\phi_{2}(s, w)<1$. On the other hand, since

$$
\left.\frac{d h\left(s, z_{2}, w\right)}{d z_{2}}\right|_{\substack{s_{2}=0 \\ z_{2}=w=1}}=\frac{b \lambda-1}{1-b \lambda_{1}}<0
$$

we conclude that $z_{2}=\phi_{2}(s, w)$ is analytic in $|w|<1$ and $s>0$ and is continuous at 
$(s, w)=(0,1)$ and that $\phi_{2}(0,1)=1$.

Now return to equation (7). Arguments similar to those used in the proof of Theorem 3 [11, page 450-452] show that the solution of differential equation (7) is given by

$$
\begin{aligned}
\psi\left(s, z_{2}, w\right) & =\int_{z_{2}}^{\phi_{2}(s, w)} \frac{\left[\phi_{1}(s, u, w)\right]^{m} u^{n}}{\nu\left[\phi_{1}(s, u, w)-u\right]} \cdot \exp \left\{\int_{u}^{z_{2}} \frac{s+\lambda-\left(\lambda-\frac{\nu}{x}\right) \phi_{1}(s, x, w)}{\nu\left[\phi_{1}(s, x, w)-x\right]} d x\right\} d u \\
& =\int_{z_{2}}^{\phi_{2}(s, w)} \frac{z_{2}\left[\phi_{1}(s, u, w)\right]^{m} u^{n-1}}{\left[\phi_{1}(s, u, w)-u\right]} \cdot \exp \left\{\int_{u}^{z_{2}} \frac{s+\nu+\lambda\left(1-\phi_{1}(s, x, w)\right)}{\nu\left[\phi_{1}(s, x, w)-x\right]} d x\right\} d u
\end{aligned}
$$

if $z_{2} \neq \phi_{2}(s, w)$.

For $z_{2}=\phi_{2}(s, w)$, we have directly from (7),

$$
\psi\left(s, z_{2}, w\right)=\frac{\left[\phi_{2}(s, w)\right]^{n+m}}{s+\nu+\lambda-\lambda \phi_{2}(s, w)}
$$

When $\left(z_{2}, w\right)=(1,1)$, note that $1 \neq \phi_{2}(s, 1)$, because $\phi_{1}(s, 1,1)=E\left[e^{-s L} \infty\right]<1$ for $s>0$. So, we will use $(8 a)$ when $\left(z_{2}, w\right)=(1,1)$.

Let $T_{m n}$ denote the time interval from the moment of the 0 -th call's departure until the tagged call occupies the server (see Figure 1). Then the Laplace-Stieltjes transform of the distribution of $T_{m n}$ is given, for $s>0$, by

$$
\begin{aligned}
& E\left[\exp \left(-s T_{m n}\right)\right]=\int_{0}^{\infty} e^{-s t} d F_{m n}(t) \\
& =\int_{0}^{\infty} P\{\text { no catastrophe occurs in }[0, t]\} d F_{m n}(t) \\
& =P\left\{\text { no catastrophe occurs in }\left[0, T_{m n}\right]\right\} \\
& =\sum_{i=0}^{\infty} \sum_{l=1}^{\infty} P\left(\text { during the period } T_{m n} \text { catlastrophe does not occur and, exactly } i\right. \\
& \text { incoming calls and } 0 \text { outgoing calls at the moment when the } \\
& \text { tagged call occupies the server) }
\end{aligned}
$$


where $F_{m n}(t)$ is the distribution function of $T_{m n}$. It should be noted that the dependence of $E\left[\exp \left(-s T_{m n}\right)\right]$ on $(m, n)$ has the form

$$
E\left[\exp \left(-s T_{m n}\right)\right]=\int_{1}^{\phi_{2}(s, 1)}\left[\phi_{1}(s, u, 1)\right]^{m} u^{n-1} f(s, u) d u
$$

where

$$
f(s, u)=\frac{1}{\phi_{1}(s, u, 1)-u} \exp \left\{\int_{u}^{1} \frac{s+\nu+\lambda\left(1-\phi_{1}(s, v, 1)\right)}{\nu\left[\phi_{1}(s, v, 1)-v\right]} d v\right\} .
$$

Finally, we find the Laplace-Stieltjes transform of the distribution of the virtual waiting time $W$ for the tagged call. Without loss of generality, we may assume that the tagged call arrives at the system at time 0 . The state of $N_{i}(t)$ at immediately prior to $t$ is denoted by $N_{i}(t-)$. Then

$$
\begin{aligned}
& E[\exp (-s W)]=E\left[e^{-s W} \mid \xi(0-)=0\right] \sum_{n=0}^{\infty} q_{n} \\
& \quad+\int_{0}^{\infty} \sum_{m=0}^{\infty} \sum_{n=0}^{\infty} E\left[e^{-s W} \mid N_{1}(0-)=m, N_{2}(0-)=n, \xi(0-)=1, X(0-)=x\right] q_{m n}(x) d x,
\end{aligned}
$$

where

$$
q_{n}=\lim _{t \rightarrow \infty} q_{n}(t), \quad q_{m n}(x)=\lim _{t \rightarrow \infty} q_{m n}(t, x)
$$

It is known [2] that the probability $\sum_{n=0}^{\infty} q_{n}$ is equal to $1-\rho$, and, for the second term of (11), we need the generating function

$$
P^{*}\left(s, z_{1}, z_{2}\right)=\sum_{m=0}^{\infty} \sum_{n=0}^{\infty}\left(\int_{0}^{\infty} e^{-s x} q_{n m}(x) d x\right) z_{1}^{m} z_{2}^{n}
$$

which can be obtained from [2, equation 4] as follows:

$$
\begin{aligned}
P^{*}\left(s, z_{1}, z_{2}\right)= & (1-\rho) \frac{\lambda\left(\beta\left(0, z_{1}, z_{2}\right)-b^{*}(s)\right)}{s-\lambda_{1}\left(1-z_{1}\right)-\lambda_{2}\left(1-z_{2}\right)} \cdot \frac{z_{1}-\phi_{1}\left(z_{2}\right)}{z_{1}-\beta\left(0, z_{1}, z_{2}\right)} \\
& \cdot \frac{1-z_{2}}{\phi_{1}\left(z_{2}\right)-z_{2}} \cdot \exp \left[-\frac{\lambda}{\nu} \int_{z_{2}}^{1} \frac{1-\phi_{1}(x)}{\phi_{1}(x)-x} d x\right]
\end{aligned}
$$

where $\phi_{1}(x)=\phi_{1}(0, x, 1)$. 
It is clear that $E\left[e^{-s W} \mid \xi(0-)=0\right]=1$ and $E\left[e^{-s W} \mid N_{1}(0-)=m, N_{2}(0-)=n\right.$, $\xi(0-)=1, X(0-)=x]$ can be rewritten in the following way

$$
\begin{aligned}
& E\left\{e^{-s W} \mid N_{1}(0-)=m, N_{2}(0-)=n, \xi(0-)=1, X(0-)=x\right\} \\
& =\exp [-(s+\lambda) x] \sum_{i=0}^{\infty} \sum_{j=0}^{\infty} \frac{\left(\lambda_{1} x\right)^{i}}{i !} \frac{\left(\lambda_{2} x\right)^{j}}{j !} E \exp \left(-s T_{m+i, n+j+1}\right) \\
& \phi_{2}(s, 1) \\
& =\int_{1} f(s, u) \exp \left[-\left(s+\lambda_{1}\left(1-\phi_{1}(s, u, 1)\right)+\lambda_{2}(1-u)\right) x\right]\left[\phi_{1}(s, u, 1)\right]^{m} u^{n} d u
\end{aligned}
$$

where we used (10) in the last inequality. Thus by (12) and (14), the second term on the right-hand side of (11) becomes

$$
\int_{1}^{\phi_{2}(s, 1)} f(s, u) P^{*}\left(s+\lambda_{1}\left(1-\phi_{1}(s, u, 1)\right)+\lambda_{2}(1-u), \phi_{1}(s, u, 1), u\right) d u
$$

Therefore we obtain from (11) and (13), that the Laplace-Stieltjes transform of the distribution of $W$ is

$$
\begin{gathered}
E[\exp (-s W)]=1-\rho+\frac{1-\rho}{s} \int_{1}^{\phi_{2}(s, 1)} \frac{\lambda(1-u)\left[\phi_{1}(0, u, 1)-\phi_{1}(s, u, 1)\right.}{\left[\phi_{1}(0, u, 1)-u\right]\left[\phi_{1}(s, u, 1)-u\right]} \\
\quad \cdot\left\{\exp \int_{u}^{1} \frac{(s+\nu)\left[\phi_{1}(0, x, 1)-x\right]+\lambda(1-x)\left[\phi_{1}(0, x, 1)-\phi_{1}(s, x, 1)\right]}{\nu\left[\phi_{1}(0, x, 1)-x\right]\left[\phi_{1}(s, x, 1)-x\right]} d x\right\} d u .
\end{gathered}
$$

In summary,

Theorem 2: $\quad$ In steady state, the Laplace-Stieltjes transform of the distribution of the virtual waiting time $W$ is given by (15), where $\phi_{1}(s, u, 1)\left(\phi_{1}(s, 1)\right.$, respectively) is the unique solution of the functional equation $\phi_{1}(s, u, 1)=b^{*}\left(s+\lambda_{1}\left(1-\phi_{1}(s, u, 1)\right)+\lambda_{2}(1-u)\right)$ $\left(\phi_{2}(s, 1)=\phi_{1}\left(s, \phi_{2}(s, 1), 1\right), \quad\right.$ respectively $)$ and satisfies the inequality $0 \leq \phi(s, u, 1) \leq 1$ $\left(0 \leq \phi_{2}(s, 1) \leq 1\right.$, respectively $)$.

Remark: When $\lambda_{1}=0$, our model becomes the ordinary $M / G / 1$ retrial queue with one type of call. In this case $\phi_{1}\left(s, z_{2}, w\right)=w b^{*}\left(s+\lambda_{2}\left(1-z_{2}\right)\right)$ and $\phi_{2}(s, w)$ is the solution of $z_{2}=w b^{*}\left(s+\lambda_{2}\left(1-z_{2}\right)\right)$. Equation (15) reduces to

$$
E[\exp (-s W)]=1-\rho+\frac{1-\rho}{s} \int_{1}^{\phi_{2}(s, 1)} \frac{\lambda(1-u)\left[b^{*}\left(\lambda_{2}-\lambda_{2} u\right)-b^{*}\left(s+\lambda_{2}-\lambda_{2} u\right)\right]}{\left[b^{*}\left(\lambda_{2}-\lambda_{2} u\right)-u\right]\left[b^{*}\left(s+\lambda_{2}-\lambda_{2} u\right)-u\right]}
$$




$$
\cdot\left\{\exp \int_{u}^{1} \frac{(s+\nu)\left[b^{*}\left(\lambda_{2}-\lambda_{2} x\right)-x\right]+\lambda(1-x)\left[b^{*}\left(\lambda_{2}-\lambda_{2} x\right)-b^{*}\left(s+\lambda_{2}-\lambda_{2} x\right)\right]}{\nu\left[b^{*}\left(\lambda_{2}-\lambda_{2} x\right)-x\right]\left[b^{*}\left(s+\lambda_{2}-\lambda_{2} x\right)-x\right]}\right\} d u
$$

which agrees with Theorem 3 in Falin [11].

\section{ACKNOWLEDGEMENT}

The authors are grateful to the referee for the valuable suggestions.

\section{REFERENCES}

[1] Alexsandrov, A.M., A queueing system with repeated orders, Engineering Cybernetics Rev. 12, 1-4, (1974).

[2] Choi, B.D. and Park, K.K., The $M / G / 1$ retrial queue with Bernoulli schedule, Queueing Systems 7, 219-227, (1990).

[3] Choo, Q.H. and Conolly, B., New results in the theory of repeated orders queueing systems, J. Appl. Prob. 16, 631-640, (1979).

[4] Cohen, J.W., Basic problems of telephone traffic theory and the influence repeated calls, Philips Telecom. Rev. 18, 49-104, (1957).

[5] Cohen, J.W., The Single Server Queue, North-Holland, 1982.

[6] Falin, G.I., Aggregate arrival of customers in one-linear system with repeated calls, Ukrainian Math. J. 28, 437-440, (1976).

[7] Falin, G.I., On the waiting time in a single-channel queueing system with secondary calls, Vestnik Moskow Univ. ser. 15. Comput. Math. Cybernet. 4, 83-87, (1977).

[8] Falin, G.I., A single-line system with secondary orders, Engineering Cybernet. 17, 7683, (1979).

[9] Falin, G.I., On the waiting time process in a single line queue with repeated calls, $J$. Appl. Prob. 23, 185-192, (1986).

[10] Falin, G.I., Survey of retrial queues, Queueing Systems 7, 127-167, (1990).

[11] Falin, G.I. and Fricker, C., On the virtual waiting time in an $M / G / 1$ retrial queue with priority customer, J. Appl. Prob. 28, 446-460, (1991).

[12] Falin, G.I., Artalejo, J.R. and Martin, M., On the single server retrial queue with priority customer, Preprint.

[13] Hashida, O. and Kawashima, K., Buffer behavior with repeated calls, Electronics and Communications in Japan 62-B, 27-35, (1979).

[14] Jaiswal, N.K., Priority Queues, Academic Press, Inc., 1988. 
[15] Khalil, Z., Falin, G.I. and Yang, T., Some analytical results for congestion in subscriber line modules, Queueing Systems 10, 381-402, (1992).

[16] Kulkarni, V.G., On queueing systems with retrials, J. Appl. Prob. 20, 380-389, (1983).

[17] Kulkarni, V.G., A game theoretic model for two types of customers competing for service, Oper. Res. Lett. 2, 119-122, (1983).

[18] Kulkarni, V.G., Expected waiting time in a multi-class batch arrival retrial queue, $J$. Appl. Prob. 23, 144-154, (1986).

[19] Kulkarni, V.G. and Choi, B.D., Retrial queues with server subject to breakdowns, Queueing Systems 7, 191-208, (1990).

[20] Yang, T. and Templeton, J.G.C., A survey on retrial queues, Queueing Systems 2, 201233, (1987). 


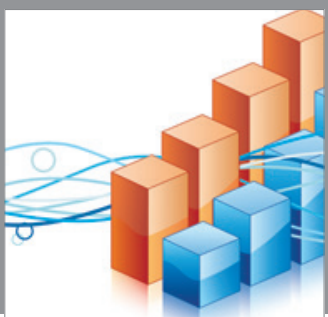

Advances in

Operations Research

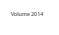

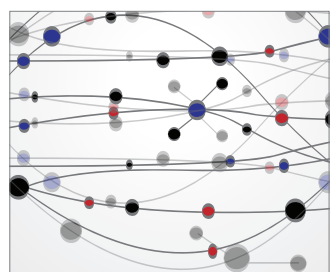

\section{The Scientific} World Journal
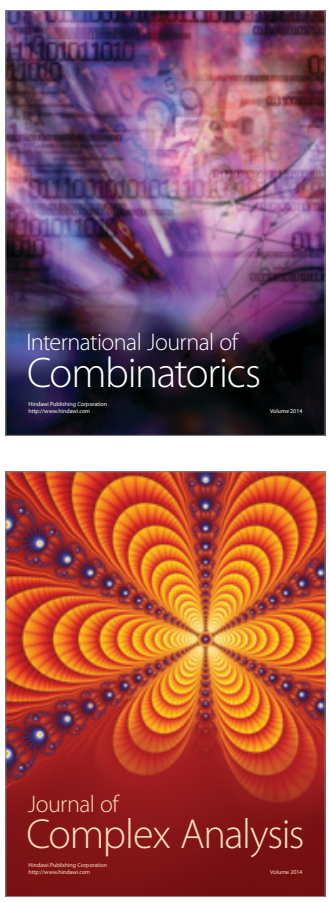

International Journal of

Mathematics and

Mathematical

Sciences
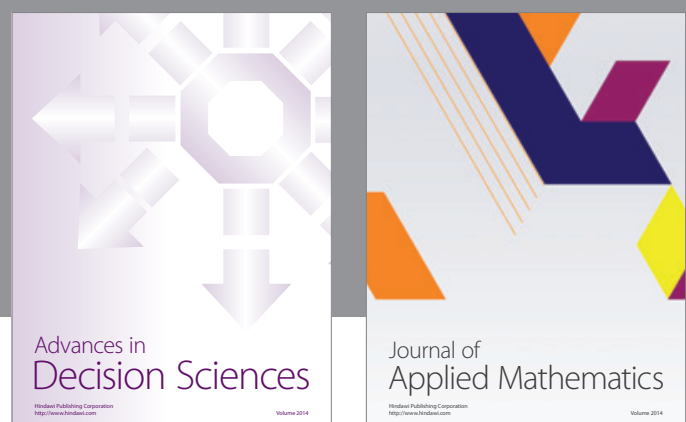

Journal of

Applied Mathematics
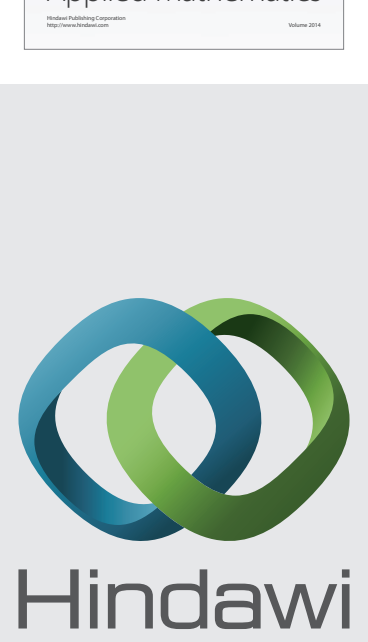

Submit your manuscripts at http://www.hindawi.com
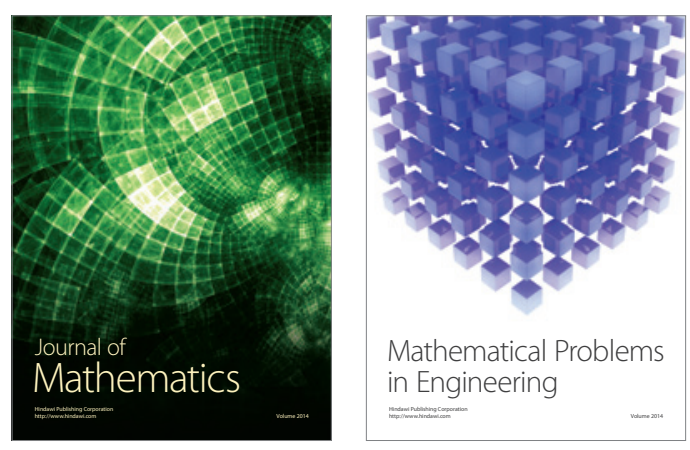

Mathematical Problems in Engineering
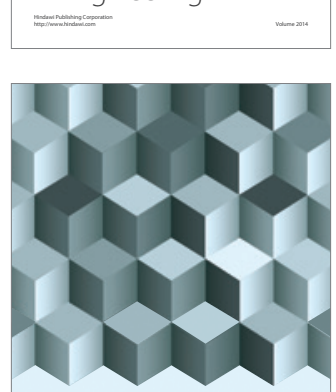

Journal of

Function Spaces
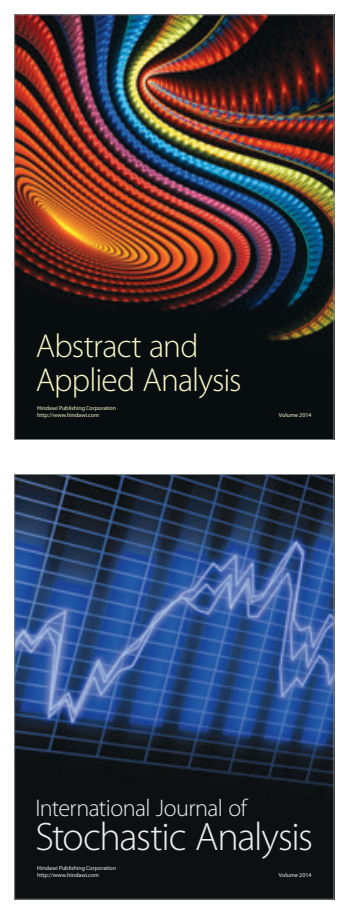

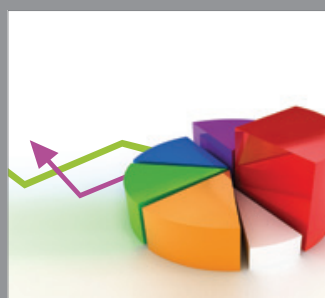

ournal of

Probability and Statistics

Promensencen
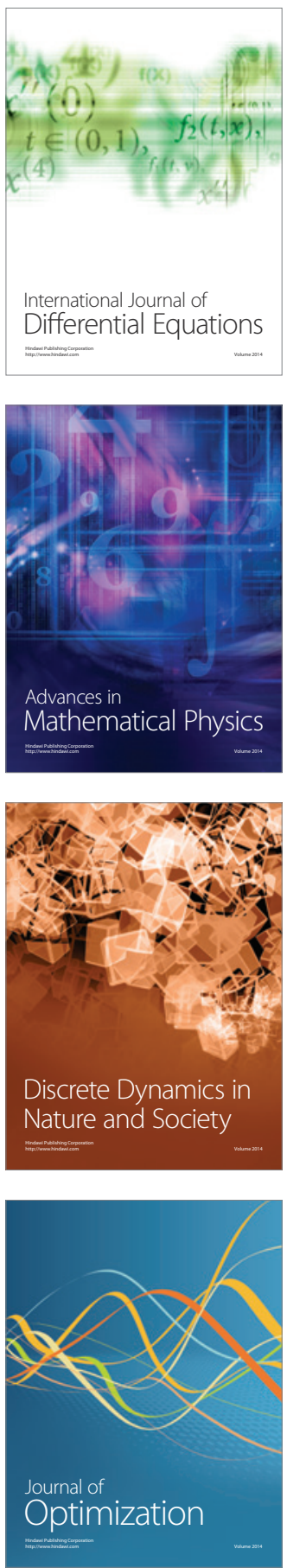